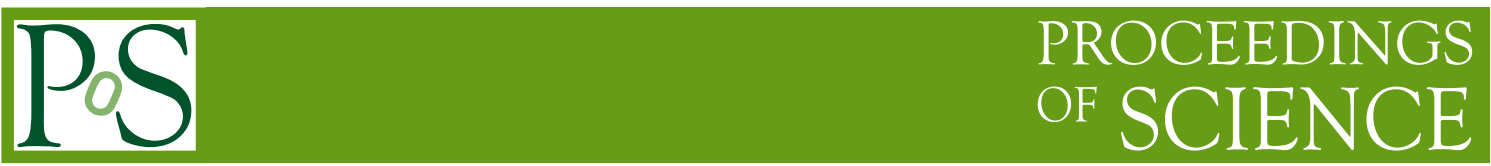

\title{
Exploring straight infinite Wilson lines towards formulating a new classical theory for gluodynamics
}

\author{
Hiren Kakkad, ${ }^{a, *}$ Piotr Kotko ${ }^{a}$ and Anna Stasto ${ }^{b}$ \\ ${ }^{a}$ AGH University Of Science and Technology, Physics Faculty, \\ Mickiewicza 30, 30-059 Krakow, Poland \\ ${ }^{b}$ The Pennsylvania State University, Physics Department, \\ 104 Davey Lab, University Park, PA 16802, USA \\ E-mail: kakkad@agh.edu.pl
}

We develop a new classical action that in addition to MHV vertices contains also $\mathrm{N}^{\mathrm{k}} \mathrm{MHV}$ vertices, where $1 \leq k \leq n-4$ with $n$ the number of external legs. The lowest order vertex is the four-point MHV vertex - there is no three point vertex and thus the amplitude calculation involves fewer diagrams than in the CSW method and, obviously, considerably fewer than in the standard YangMills action. The action is obtained by a canonical transformation of the Yang-Mills action in the light-cone gauge, where the field transformations are based on Wilson line functionals.

*** The European Physical Society Conference on High Energy Physics (EPS-HEP2021), ***

*** 26-30 July 2021 ***

*** Online conference, jointly organized by Universität Hamburg and the research center DESY ***

${ }^{*}$ Speaker 


\section{Introduction}

The following work focuses on a description of pure gluonic scattering amplitudes in terms of a new action, currently developed at the classical level (thus suitable for tree amplitudes). Despite considered as fundamental, gluon fields are often not the most efficient degrees of freedom for computing amplitudes. Interestingly, in [1, 2], the Maximally Helicity Violating (MHV) vertices used in the Cachazo-Svrcek-Witten (CSW) method [3] were shown to be connected with straight infinite Wilson lines on the self-dual plane. Following that, in [4], we derived a new classical action for gluodynamics in which the fields are directly related to Wilson line functionals. The new action does not have the triple-gluon vertices at all. This is because the triple-gluon vertices have been effectively resummed inside the Wilson lines. Higher-point vertices include not only the MHV vertices, but also other helicity configurations. The number of diagrams needed to obtain amplitudes beyond the MHV level is thus greatly reduced. We performed explicit calculations within the new formulation of several higher multiplicity amplitudes, to verify the consistency of the results.

\section{Self-Dual Yang-Mills theory and scattering amplitudes}

The starting point is the full Yang-Mills action on the constant light-cone time $x^{+}$in the light-cone gauge $\hat{A}^{+}=0$ (we denote $\hat{A}=A_{a} t^{a}$ ). Integrating out the $\hat{A}^{-}$fields (appearing quadratically) from the partition function, leaves only two complex fields $\hat{A}^{\bullet}, \hat{A}^{\star}$ that correspond to plus-helicity and minus-helicity gluon fields. The action in this setup has $(++-),(--+)$ and $(++--)$ vertices. The fully covariant form of the self dual equations is $\hat{F}^{\mu \nu}=* \hat{F}^{\mu \nu}$ where the Hodge dual is defined as $* \hat{F}_{\mu \nu}=-i \epsilon_{\mu \nu \alpha \beta} \hat{F}^{\alpha \beta}$. The corresponding self dual equation in light-cone gauge, can be obtained from the truncated action consisting of just the kinetic term and the $(++-)$ vertex.

We are interested in the classical solution to the self-dual EOM that give us information about scattering amplitudes. The tree-level Green functions can be extracted by coupling the classical action to an external current $\hat{j}$ and postulating the power series solution $A_{a}^{\bullet}[j](x)$. Assuming the currents $\hat{j}$ are supported on the light-cone, the momentum space off-shell currents generated by the solution correspond to the off-shell currents similar to the Berends-Giele currents, i.e. to the amplitude of an off-shell gluon scattering into on-shell gluons.

\section{Straight infinite Wilson lines in Self-Dual and MHV Lagrangians}

It is very interesting, how the self-dual equation encodes an infinite Wilson line spanning over the transverse complex plane [2]. This can be simply demonstrated as follows. First assume a power series expansion for the inverse functional $j_{a}\left[A^{\bullet}\right]$. The kernels for this expansion can be found by substituting it to $A_{a}^{\bullet}[j](x)$. Then, it is straightforward to realise that the kernels are nothing but the momentum space expansion coefficients of the following straight infinite Wilson line (in the light-cone gauge) [1]:

$$
j_{a}[A](x)=\int_{-\infty}^{\infty} d \alpha \operatorname{Tr}\left\{\frac{1}{2 \pi g} t^{a} \partial_{-} \mathbb{P} \exp \left[i g \int_{-\infty}^{\infty} d s \varepsilon_{\alpha}^{+} \cdot \hat{A}\left(x+s \varepsilon_{\alpha}^{+}\right)\right]\right\},
$$

where $\varepsilon_{\alpha}^{ \pm}=\varepsilon_{\perp}^{ \pm}-\alpha \eta$. Notice, that this four vector has the form of a gluon polarization vector. Indeed for $\alpha=p \cdot \varepsilon_{\perp}^{ \pm} / p^{+}$, it is the transverse polarization vector for a gluon with momentum $p$. Thus, in 


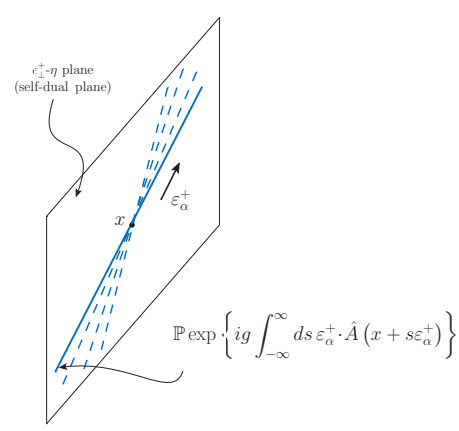

(a)

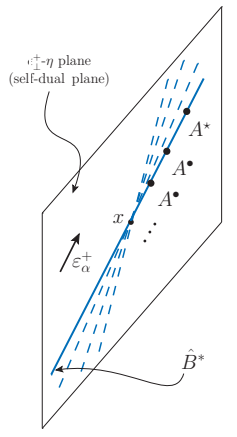

(b)

Figure 1: Left: The straight infinite Wilson line lying on the plane spanned by $\varepsilon_{\alpha}^{+}=\varepsilon_{\perp}^{+}-\alpha \eta$ and integrated over all $\alpha$ (the dashed lines represent tilted Wilson lines due to the change of $\alpha$ ). Right: The $B^{\star}$ field can be represented as the straight infinite Wilson line similar to the one on the left, but where one $A^{\bullet}$ field has been replaced by the $A^{\star}$ field in the functional expansion (with a suitable symmetry factor).

momentum space the Wilson line lies along the plus helicity polarization vector. Interestingly, the two vectors defining the direction of the Wilson line, $\varepsilon_{\perp}^{+}$and $\eta$, span the so-called self-dual plane (the plane on which the tensors are self-dual). Note, however, that the Wilson line is integrated over all possible directions $\alpha$ on the self-dual plane gaining a projective character (see Fig. 1a).

The Wilson line (1) has been already recognized in [1] as the relation between the positive helicity Yang-Mills field and positive helicity field in the action [5] (the so called 'MHV action') implementing the CSW rules [3]. Briefly, the MHV action is obtained from the Yang-Mills action by canonically transforming both the fields $\hat{A}^{\bullet}, \hat{A}^{\star}$ to a new pair of fields $\left(\hat{B}^{\bullet}, \hat{B}^{\star}\right)$ with a requirement that the kinetic term and the $(++-)$ triple-gluon vertex is mapped to the kinetic term in the new action. In the original work [5] the MHV action was constructed using only analytic properties of the transformations and equivalence theorem for the S-matrix. The explicit solution for $\hat{A}^{\bullet}$ and $\hat{A}^{\star}$ fields was found in [6] in momentum space. The Wilson line interpretation of the new fields in the MHV action was first discussed in [1,2]. The plus helicity field, $B_{a}^{\bullet}\left[A^{\bullet}\right](x)$, was shown in [1] to be the straight infinite Wilson line (1). On the other hand, the minus helicity field, $B_{a}^{\star}\left[A^{\bullet}, A^{\star}\right](x)$ was shown in [2] to be a similar Wilson line, but with an insertion of the minus helicty gluon field at certain point on the line (see Fig. 1b). Since it is natural to think about the $A^{\star}$ fields as belonging to Wilson lines living within the anti-self-dual plane spanned by $\varepsilon_{\alpha}^{-}$and $\eta$ (recall that the $B^{\bullet}$ lives on the plane spanned by $\varepsilon_{\alpha}^{+}$), we conjectured in [2] that the solution $B_{a}^{\star}\left[A^{\bullet}, A^{\star}\right](x)$ should just be a cut through a bigger structure, spanning over both planes.

\section{A new Wilson-Line based action for Gluodynamics}

The Mansfield's transformation [5] eliminates one of the triple gloun vertex $(++-)$ while the other triple gloun vertex $(+--)$ still exists in the MHV action. Triple point vertices are not very effective building blocks for calculating amplitudes, and, actually they do not constitute any physical amplitude themselves - in the on-shell limit they are zero (for real momenta). Motivated by the geometric considerations mentioned earlier and the above arguments, we proposed in [4] a more general transformation $\left\{\hat{A}^{\bullet}, \hat{A}^{\star}\right\} \rightarrow\left\{\hat{Z}^{\bullet}\left[A^{\bullet}, A^{\star}\right], \hat{Z}^{\star}\left[A^{\bullet}, A^{\star}\right]\right\}$ leading to a new action. These transformations are based on path ordered exponentials of the gauge fields, extending over both the self-dual and anti-self-dual planes. It maps the kinetic term and both the triple-gluon vertices of the Yang-Mills action into a free term in the new action. In order to preserve the functional measure in the partition function, up to a field independent factor, it is necessary that the transformation is 
canonical. The transformation can be solved to obtain the explicit solutions for $\hat{Z}^{\bullet}\left[A^{\bullet}, A^{\star}\right]$ and $\hat{Z}^{\star}\left[A^{\bullet}, A^{\star}\right]$ fields [4]. Substituting their inverse in the Yang-Mills action results in the new action. For convenience, we shall call the new action as Z-field action hereafter. It has the following generic structure:

$$
\begin{aligned}
S_{\mathrm{Y}-\mathrm{M}}^{(\mathrm{LC})}\left[Z^{\bullet}, Z^{\star}\right]=\int d x^{+}\left[\mathcal{L}_{-+}^{(\mathrm{LC})}\right. & +\mathcal{L}_{--++}^{(\mathrm{LC})}+\mathcal{L}_{--+++}^{(\mathrm{LC})}+\mathcal{L}_{--++++}^{(\mathrm{LC})}+\ldots \\
& +\mathcal{L}_{---++}^{(\mathrm{LC})}+\mathcal{L}_{---+++}^{(\mathrm{LC})}+\mathcal{L}_{---++++}^{(\mathrm{LC})}+\ldots \\
& \vdots \\
& \left.+\mathcal{L}_{---\ldots-++}^{(\mathrm{LC})}+\mathcal{L}_{---\ldots-+++}^{(\mathrm{LC})}+\mathcal{L}_{---\ldots-+++}^{(\mathrm{LC})} \ldots \ldots\right],
\end{aligned}
$$

The key properties of the action are:

$i$ ) There are no three point interaction vertices. The reason is that the triple-gluon vertices have been effectively resummed inside the Wilson lines.

ii ) At the classical level there are no all-plus, all-minus, as well as $(-+\cdots+),(-\cdots-+)$ vertices.

iii ) There are MHV vertices, $(--+\cdots+)$, corresponding to MHV amplitudes in the on-shell limit.

iv ) There are $\overline{\mathrm{MHV}}$ vertices, $(-\cdots-++)$, corresponding to $\overline{\mathrm{MHV}}$ amplitudes in the on-shell limit.

v) All vertices have the form which can be easily calculated. In fact we reported a compact general form for any vertex in the Z-field action in [4].

Using this new action we computed several tree-level amplitudes. For example, for the 6-point Next-To-MHV (NMHV) amplitude with helicity configuration $(---+++)$ we have just three contributing diagrams. The sum of these diagrams reproduce in the on-shell limit the known result [7]. For 7-point NNMHV amplitude $(----+++)$ we had just five contributing diagrams depicted in Fig. 2. Furthermore, the higher multiplicity amplitudes, up to 8-point NNMHV $(----++++)$,

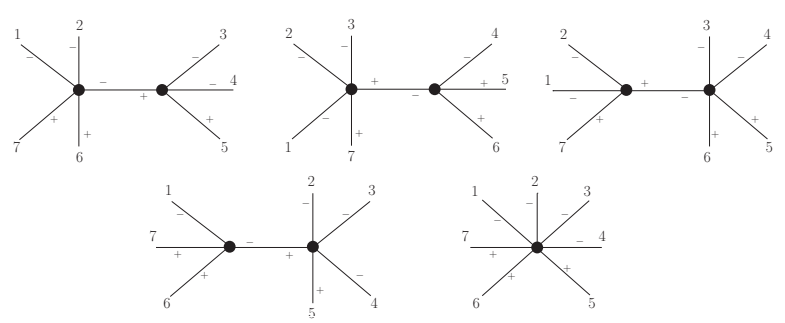

Figure 2: Diagrams contributing to the 7-point NNMHV amplitude $(----+++)$.

were calculated and shown to be in agreement with the standard methods [8]. The maximum number of diagrams we encountered in that case was 13. The absence of triple-gluon vertices resulted in fewer diagrams required to compute amplitudes, when compared to the CSW method and, obviously, considerably fewer than in the standard Yang-Mills action.

\section{Acknowledgements}

H.K. and P.K. are supported by the National Science Centre, Poland grant no. 2018/31/D/ST2 /02731. A.M.S. is supported by the U.S. Department of Energy Grant DE-SC-0002145 and in part by National Science Centre in Poland, grant 2019/33/B/ST2/02588. 


\section{References}

[1] P. Kotko and A.M. M Stasto. Wilson lines in the MHV action. J. High Energy Phys., 2017(9):47, 2017.

[2] Hiren Kakkad, Piotr Kotko, and Anna Stasto. Exploring straight infinite Wilson lines in the self-dual and the MHV Lagrangians. Phys. Rev. D, 102(9):094026, nov 2020.

[3] Freddy Cachazo, Peter Svrcek, and Edward Witten. MHV Vertices And Tree Amplitudes In Gauge Theory. J. High Energy Phys., 2004(09):006-006, sep 2004.

[4] Hiren Kakkad, Piotr Kotko, and Anna Stasto. A new wilson line-based action for gluodynamics. Journal of High Energy Physics, 2021(7), Jul 2021.

[5] Paul Mansfield. The lagrangian origin of MHV rules. J. High Energy Phys., 2006(03):037-037, mar 2006.

[6] James H Ettle and Tim R Morris. Structure of the MHV-rules lagrangian. J. High Energy Phys., 2006(08):003-003, aug 2006.

[7] David A Kosower. Light-cone recurrence relations for QCD amplitudes. Nucl. Phys. B, 335(1):23-44, apr 1990.

[8] Lance J Dixon, Johannes M Henn, Jan Plefka, and Theodor Schuster. All tree-level amplitudes in massless QCD. J. High Energy Phys., 2011(1):35, jan 2011. 